\title{
A SMART INTERNET-OF-THINGS APPLICATION FOR SHOE RECOMMENDATIONS USING PRESSURE SENSOR AND RASPBERRY PI
}

\author{
Yutian Fan $^{1}$, Yu Sun ${ }^{2}$ and Fangyan Zhang ${ }^{3}$ \\ ${ }^{1}$ Milton Academy, Milton, MA, 02186, USA \\ ${ }^{2}$ Department of Computer Science California State Polytechnic University, \\ Pomona, CA, 91768, USA \\ ${ }^{3}$ ASML, San Jose, CA, 95131, USA
}

\begin{abstract}
Running is one of the most important and simple sports spanning various ages, which can train throughout body and muscle. For running, proper shoes not only improve runners' performance but also protect them from injury to some extent. However, runners have difficulty in finding a pair of shoes which fit runners' gait patterns and feet shape very well. The process of selection of shoes is not effective and necessarily accurate. In this paper, we propose a new tool which facilitates the process by employing electronic sensors to the insoles of shoes and collecting feet information for runner accurately. It is helpful for runners to find the best fit shoes.
\end{abstract}

\section{KEYWORDS}

Machine learning, Firebase, Mobile application, Model fitting

\section{INTRODUCTION}

\subsection{The Importance of Shoes}

Many say that running is a sport that little to no requirement for equipment: all that one needs is a pair of shoes [1][2]. However, this one pair of shoes can have an immense long-term impact to the runner. Shoes that don't fit the runner can cause injuries varied from average shin splints to achilles tendonitis and insertional achilles tendinopathy. Other than preventing health risks, a fitting pair of training shoes can also increase the speed of a runner. Different models of shoes targets to support different kinds of feet, and the right kind of support can provide the runner better balances and lighter strides [3][4]. Competitive athletes, even in middle schools, are suggested to buy a new pair of shoes every year to avoid the disadvantages from overused, unbalanced shoe sole. Professionals always have multiple pairs of customized shoes to maximize the result of both their training and competing.

\subsection{Current Ways of Shoe Picking}

Currently, a lot of non-competitive runners simply go to sports stores such as Dicks and purchase whatever shoes that has appealing appearance. Often, these runners get shoes that don't actually fit with their gait patterns and feet shape. They either suffer from injuries previously mentioned or simply can't reach their full potential. Competitive runners usually go to stores like marathon sports and consults the staff there to get the right shoe model. However, the process is time David C. Wyld et al. (Eds): MLNLP, BDIoT, ITCCMA, CSITY, DTMN, AIFZ, SIGPRO - 2020 pp. 137-142, 2020. CS \& IT - CSCP 2020 
consuming and not necessarily accurate. The staff looks at the runner's feet with different shoes on, asks about the runner's events or distances, and then suggest the runner several models for their subjective opinion on the comfort level. The shoe-picking process is good but can be betterthe staff can't really see the foot or feel the pressure applied to the shoes directly.

\subsection{Goals of This Project}

This project aims to improve the shoe selecting process by employing electronic sensors to the insoles of shoes to collect all the data needed to judge the fitting model for a runner. The shoes picked according to these data can be the best fits for the runners and maximizes both their comfort and their performance during practices and competitions.

The project has 4 main components: The Raspberry Pi, the Firebase [5][6], the Repl server and the mobile app.

The rest of the paper is organized as follows: Section 2 gives the details on the challenges that we met during the experiment and designing the sample; Section 3 focuses on the details of our solutions corresponding to the challenges that we mentioned in Section 2; Section 4 presents the related work. Finally, Section 5 gives the conclusion remarks, as well as pointing out the future work of this project.

\section{Challenges}

\subsection{The Hardware}

The shoe insoles are going to take the full weight of the user's body, therefore where to put what kind of sensors is the first question. Currently, the project contains a Raspberry Pi and the breadboard full of circuits. Both the Pi and the breadboard are too big to be in a shoe. The size of the wire can be solved by making customized chips, but the alternative for the Raspberry Pi is not yet find.

\subsection{The Software}

There are a few requirements for the current software to function properly: 1, The Raspberry Pi [7][8] has to be connected to the WIFI for it to be able to send data to the Firebase. 2, The Firebase has to be checked and free of excessive data. 3, The Repl server has to be on and running (if the browser tab is closed, the server can't run). These requirements are easy to satisfy when the product is used by only one person. When the number of users increases, parts such as the Firebase and the Repl server needs better alternatives to support large quantity of access. 


\section{Solutions}

\subsection{Overview of the Solution}

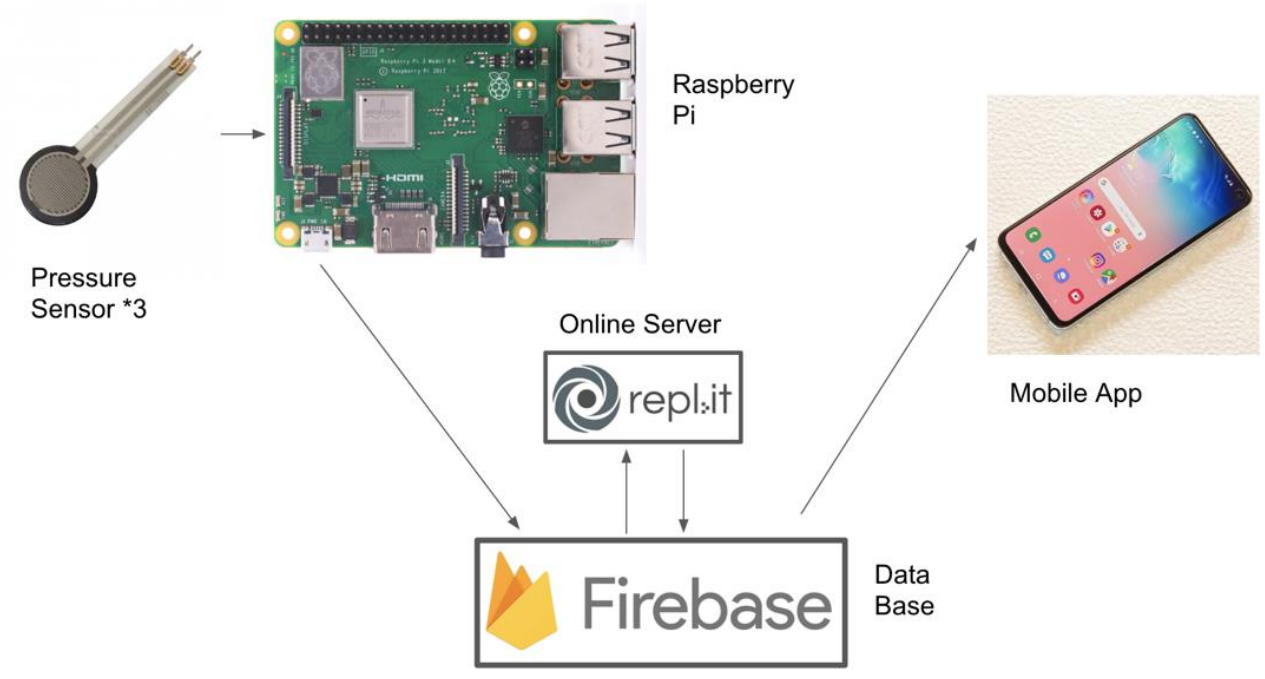

Figure 1: Overview of the solution

\subsection{The Raspberry PI}

The Raspberry $\mathrm{Pi}$ is the main hardware of this project. Currently, 3 pressure sensors are connected to the Pi via breadboard. The more pressure that are applied to the sensors, the more frequently the sensors send signals to the Pi. The Pi is programmed to calculate the timespan between each signal and therefore judge the pressure that is applied to the sensors. Then, the Pi sends the calculated pressure to the Firebase, an online Database.

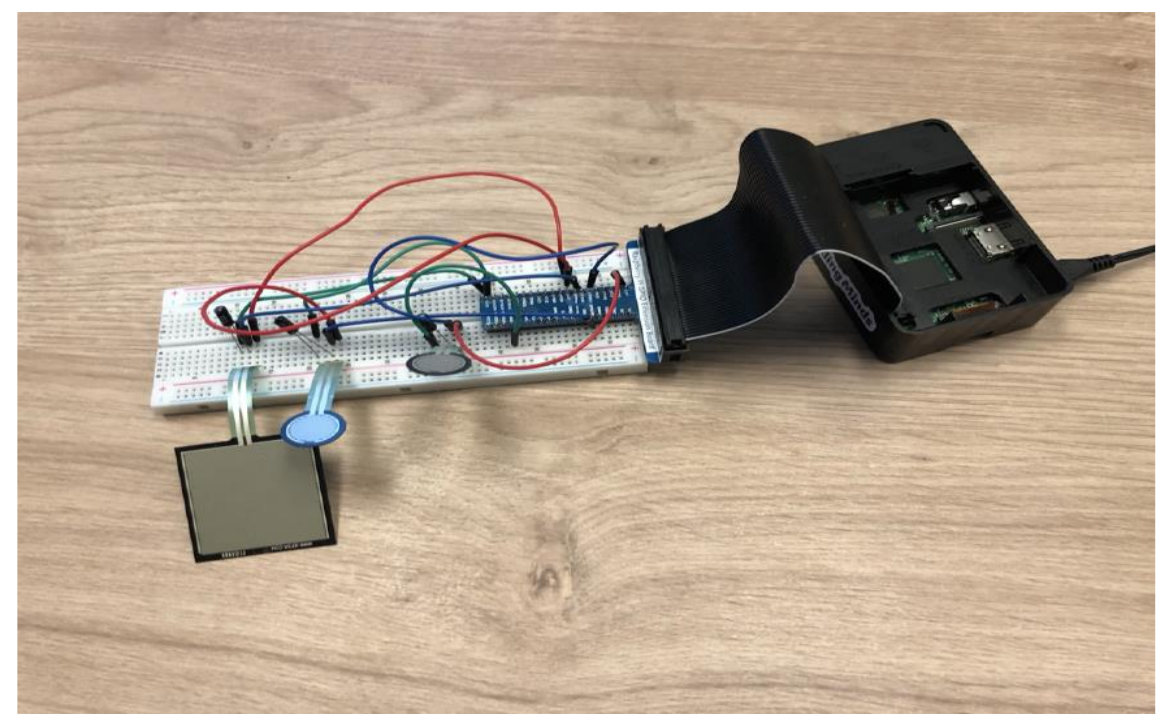

Figure 2: Hardware of Raspberry Pi 


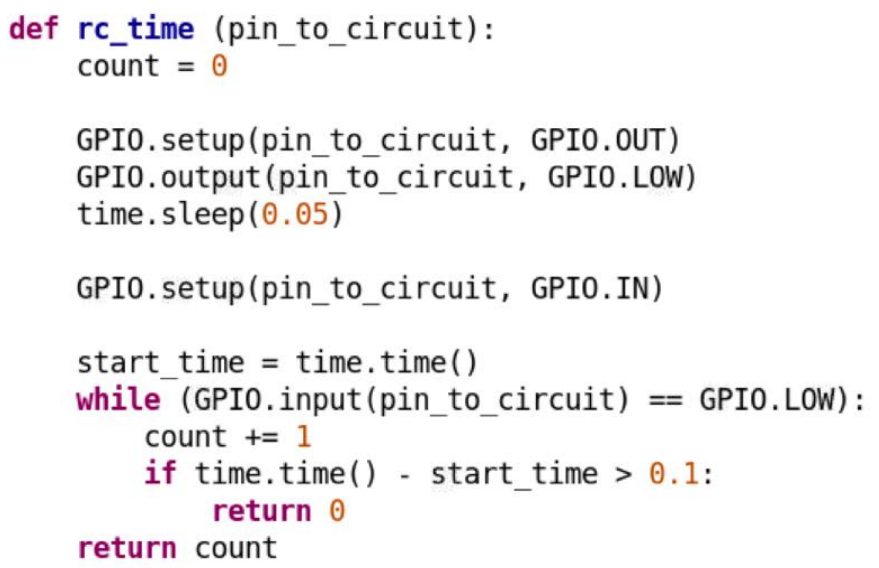

Figure 3: Code to get reading from one sensor

\subsection{The REPL Server}

The Repl is the online server, and most of the calculation happens here. The raw data in the Firebase are extracted and converted to average pressure and stride lengths. These data can be used to determine where the pressure point of the foot is, and which model is to the most suitable for the foot shape. After the calculations, Repl sends this information back to Firebase to store.

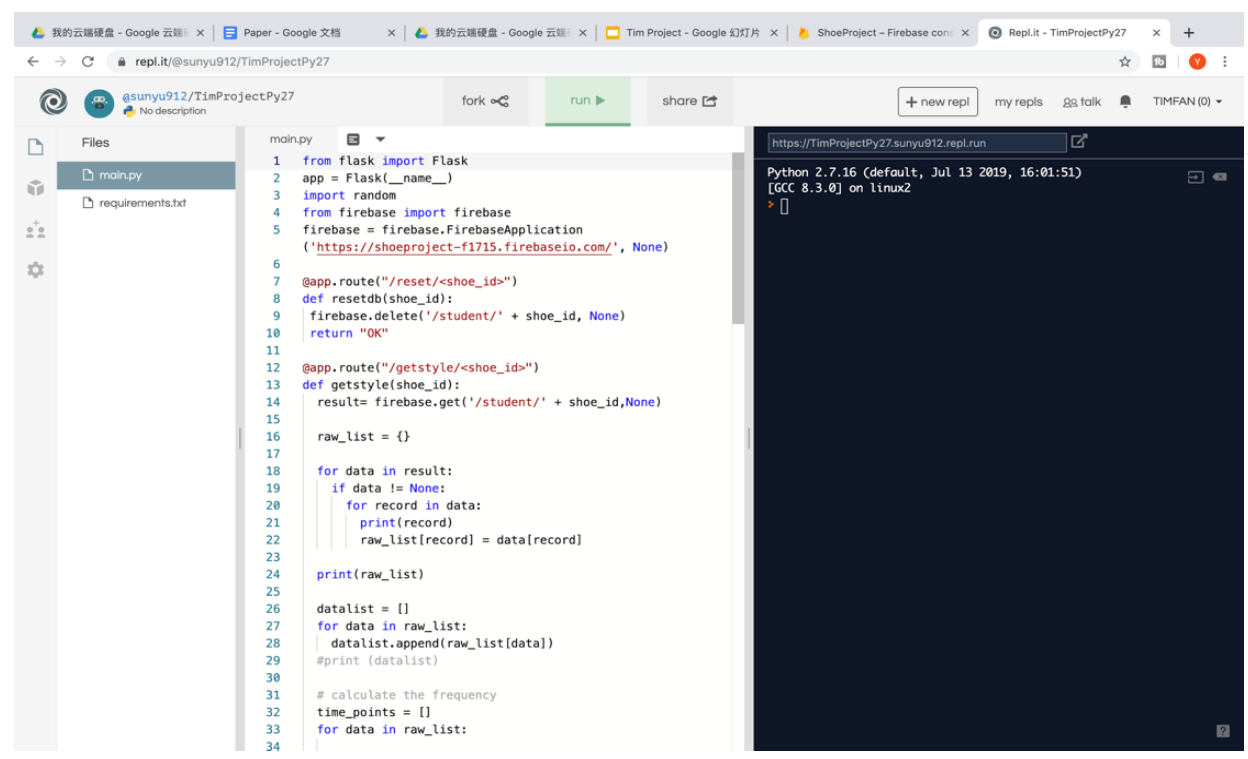

Figure 4: Overview of Repl Server running

\subsection{The Mobile Application}

The Mobile App [9][10] is the direct connection between this system and the users. The users, after uploading their data from the smart insole, can access their data by imputing the serial number of the insole. Then the mobile app extracts all the information calculated by Repl from the Firebase and presents the information to the user. The information includes and not limited to: the average pressure on each sensor, the stride frequency and the suggested model. 


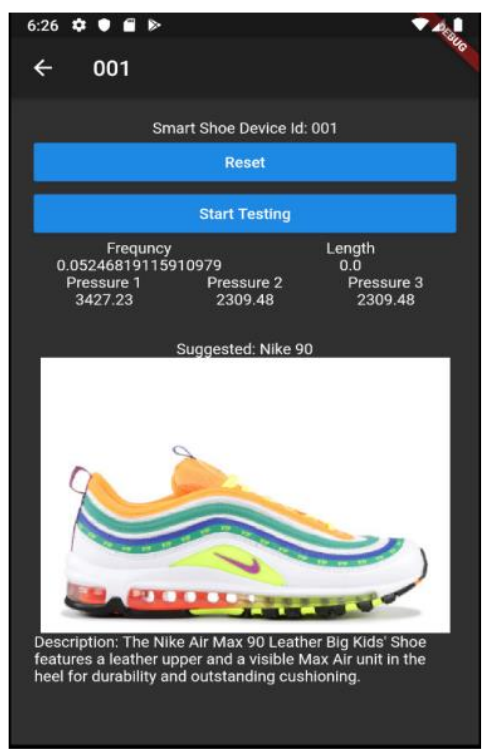

Figure 5: Screenshot of Mobile App

\section{RELATED WORK}

Smart sole [11] designed its product for a wide range of uses and people. The shoes include health analytics, smart connectivity, and sneaker design. The shoes have movement sensors to analyze pronation, supination, propulsion levels, impact force, fatigue, posture, steps, calories, and more creating precise data that prevents injuries and improves the user's health. The shoes can be connected via Bluetooth and a multi-function mobile app. The shoes have auto-lacing, temperature regulation with heating, and more. The Smartshoe is designed into an "ultra-light, premium leather and Neotech EVA shoe".

The shoes have pressure sensors in the soles that sense when to put the foot inside and triggers an algorithm that allows an automatic lacing. With integrated LEDs, the shoes can alert a user of low battery or a tight fit. Moreover, these shoes do not need charging every day, and the charge can last up to two weeks.

HOVR Phantom and HOVR Sonic [12] shoes released in February 2018 by Under Armour have inbuilt sensors to record a number of metrics important for runners. These include pace, distance, steps, stride, and cadence. These chip-laden shoes can be easily synced to the Map My Run app and are compatible with iOS [9] and Android [8] phones. The users can experience zero gravity and a great energy run with the shoes' excellent cushioning properties and comfort.

These designs are advanced smart shoes. They are created by big company with professional scientists and designers. However, most of these products are not for athletic uses. And each of these shoes still has just one model. They just can't possibly fit everyone's feet. The Smart Sole project aims not to create the best pair of shoes but the best way for the users to find their own best fit shoes. As for shoe picking, nobody has yet to make a successful and popular product.

\section{Conclusion}

Currently, the Smart Sole project is nothing but an experiment and a prototype. The size of the hardware needs to be shrunk down and the software needs to be upgraded for public use. However, the potential of this project is immense. People don't need to create the perfect shoe. 
There are so many models or running shoes in the world that hardly anyone don't have a fit. The tricky part is to find that right fit for everyone. Smart Sole aim to solve this problem and link the runners to the best shoes they never had. If Smart Sole is proved to be successful, it can benefit all the runners in the world. While, this project has a lot of space for improvement. I plan to take some steps forward in the following fields:

$>$ Find a replacement for the Repl server.

$>$ Shrink down the size of the hardware, definitely replacing the breadboard with chip and potentially replacing the Raspberry Pi.

$>$ Make shoes in a pair, measure and separate the data from both feet.

$>$ Make the pairing of the shoe and Wi-Fi connecting easier for none-coders.

\section{ACKNOWLEDGEMENTS}

I would like to give special thanks to Doctor Yu Sun, who introduced me and taught me a lot of ways to utilize Flutter, Repl, and Firebase. The hours of my research time in his university lab and his kind inspiration made this project possible. I am also grateful to FangyanZhangand Dylan Lazar for helping me with the circuit building and Pi software testing. Also, thanks to Mr. Chris Hales for providing me with great support while I was doing my further research of this project in my school (Milton Academy). I was able to build this project from scratch only because of their help.

\section{REFERENCES}

[1] Young, W. B., R. James, and I. Montgomery. "Is muscle power related to running speed with changes of direction?" Journal of Sports Medicine and Physical Fitness 42, no. 3 (2002): 282-288.

[2] McKenzie, D. C., D. B. Clement, and J. E. Taunton. "Running shoes, orthotics, and injuries." Sports medicine 2, no. 5 (1985): 334-347.

[3] Wezel, Frank V., and Terry Mackness. "Running shoes." U.S. Patent 4,624,061, issued November 25, 1986.

[4] Richards, Craig E., Parker J. Magin, and Robin Callister. "Is your prescription of distance running shoes evidence-based?." British journal of sports medicine 43, no. 3 (2009): 159-162.

[5] Alsalemi, Abdullah, Yahya Al Homsi, Mohammed Al Disi, Ibrahim Ahmed, FaycalBensaali, Abbes Amira, and Guillaume Alinier. "Real-time communication network using firebase cloud IoT platform for ECMO simulation." In 2017 IEEE International Conference on Internet of Things (iThings) and IEEE Green Computing and Communications (GreenCom) and IEEE Cyber, Physical and Social Computing (CPSCom) and IEEE Smart Data (SmartData), pp. 178-182. IEEE, 2017.

[6] Ferdoush, Sheikh, and Xinrong Li. "Wireless sensor network system design using Raspberry Pi and Arduino for environmental monitoring applications." Procedia Computer Science 34 (2014): 103-110.

[7] Upton, Eben, and Gareth Halfacree. Raspberry Pi user guide. John Wiley \& Sons, 2014.

[8] Butler, Margaret. "Android: Changing the mobile landscape." IEEE Pervasive Computing 10, no. 1 (2010): 4-7.

[9] Seabrook, Heather J., Julie N. Stromer, Cole Shevkenek, AleemBharwani, Jill de Grood, and William A. Ghali. "Medical applications: a database and characterization of apps in Apple iOS and Android platforms." BMC research notes 7, no. 1 (2014): 573.

[10] Janssen, Mark, JeroenScheerder, Erik Thibaut, AarnoutBrombacher, and Steven Vos. "Who uses running apps and sports watches? Determinants and consumer profiles of event runners' usage of running-related smartphone applications and sports watches." PloS one 12, no. 7 (2017): e0181167.

[11] Mial, Yurri. "Shoe sole." U.S. Patent Application 29/664,224 filed May 14, 2019.

[12] What It's Like To Run In Under Armour's HOVR Sonic Running Shoes https://www.besthealthmag.ca/best-you/running/hovr-sonic/ 